\title{
CLIMA FAMILIAR Y LA AUTOESTIMA DE LOS ESTUDIANTES DEL CUARTO Y QUINTO GRADO DE SECUNDARIA DEL COLEGIO CHAMPAGNAT DE TACNA 1999.
}

Ruth Salinas Lunario'; Hernan Rojas Moscoso $o^{2}$ Mario Matos Peña ; Lourdes Zegarra Salinas ${ }^{4}$

\section{RES UMEN}

¿Cómo es la relación del clima familiar y la autoestima de los estudiantes del cuarto y quinto año de secundaria del Colegio Nacional Champagnat de Tacna, 1999? Cuyo Objetivo es establecer las relaciones que existen entre el clima social familiar y la autoestima de los hijos. Es un estudio descriptivo correlacional, con el diseño de correlaciones, con una muestra de 126 familias, todos varones de los cuales 71 son de cuarto grado y 55 de quinto grado de secundaria.

Los instrumentos de recolección de datos que se utilizaron fue por encuesta y por la aplicación del inventario de autoestima de Coopersmith, y la aplicación de la escala Clima Social Familiar de Moos.

Los resultados fueron calculados según el promedio de desviación estándar, moday coeficiente de correlación de Pearson.

En conclusión la mayoria de las familias están compenetradas, se apoyan entre sí, se estimulan a actuar libremente y a expresar sus sentimientos.

Existe un moderado grado de valoración a la autoestima, a la forma de decisiones propias, al interés por las actividades intelectuales sociales y culturales.

El valor de participación en las actividades recreativas sor poco significativas. actividades.

La mayoria de las familias le dan gran importancia a la organización y planificación de sus

Existe un escaso grado de dirección familiar en función a reglas y procedimientos establecidos.

La autoestima de los estudiantes, en su mayoría presentan una autoestima normal, se evidencia por su aceptación y valoración; perciben ser importantes en su hogar y en el colegio.

\section{A B S T R A C T}

How it is the relation of the familiar climate and the self-esteem ofthe students of the quarter and fifth year of secondary of theNational School Champagnat of Tacna, 1999? Whose Objective is to establish the relations that exist between thefamiliar climate social and the self-esteem of the children. It is a corelational descriptive study, with the design of correlations, with a sample of 126 families, all men of which 71 is of fourth degree and 55 of fifth degree ofsecondary.

The instruments of data collection that was used were by survey andthe application of the inventory of self-esteem of Coopersmith, and the application of the scale Familiar Social Climate of Moos.

The results were calculated according to the average of standarddeviation, fashion and coefficient of correlation of Pearson.

In conclusion most of the families they are compenetradas, they lean between if, they are stimulated to act freely and to express its feelings.

\footnotetext{
(1) Magister en Enfermería

(2) Psicólogo

(3) Licenciado en Educación

(4) Estudiante de Enfermeria
} 
A moderate degree of valuation to the self-esteem exists, to the form of own decisions, to the interest by the social and cultural intellectual activities.

The value of participation in the recreational activities is littlesignificant. their activities.

Mayoria of the families gives to great importance to the organizationand planning him of

It exists a little degree of familiar direction in function of rulesand established procedures.

The self-esteem of the students, in its majority they present/display a normal self-esteem, one demonstrates by its acceptance and valuation; that they perceive to be important in its home and the school.

\section{INTRODUCCIÓN}

Los diferentes tipos de personalidad que reflejan los jóvenes son resultado de la interacción del niño con sus padres y otros adultos significativos para él, las influencias de los padres tienen sus efectos desde el comienzo de la vida, de tal modo que la personalidad del niño comienza a formarse desde los primeros momentos de interacción familiar.

La forma en que los padres muestran su amor hacia sus hijos, es fundamental para determinar el desarrollo afectivo de éstos, el sentimiento del niño de que es valioso como persona, constituye el centro de su personalidad y determina el uso que haga de sus habilidades, capacidades y aptitudes.

Una familia con un clima saludable es aquella que estimula el crecimiento de sus miembros, y por tanto, de autoestima, es decir, los hacen sentir personas capaces de todo, llenas de energla y bienestar, seguras de que son importantes.

\section{PROBLEMA}

¿Cómo es la relación del clima familiar y la autoestima de los estudiantes de cuarto y quinto grado de secundaria del Colegio Champagnat de Tacna 1999 ?

\section{OBJETIVOS}

- Establecer las relaciones que existen entre el clima social familiar y la autoestima de los hijos.

- Categorizar y analizar el clima social familiar en las familias de los estudiantes seleccionados.

- Diagnosticar la autoestima de los estudiantes del cuarto y quinto grado de secundaria del colegio Champagnat.

- Relacionar las variables clima social familiar y la autoestima de los hijos de las familias de la muestra.

\section{HIPÓTESIS}

Existe una relación directa entre el clima social familiar y la autoestima de los hijos del cuarto y quinto grado de secundaria del Colegio Champagnat.

\section{METODOLOGIAA}

Estudio descriptivo correlacional con diseño de correlaciones con una muestra poblacional de 162 familias y 162 alumnos que cursan el cuarto y quinto grado de secundaria.

\section{RECOLECCIÓN DE DATOS}

Elaboración de una encuesta para seleccionar a estudiantes de cuarto y quinto de secundaria y a las familias de estos.

Aplicación del inventario de Autoestıma de Coopersmith.

Aplicación de la escala de Clima social familiar de Moos a los padres seleccionados.

Descripción de los instrumentos.

\section{PROCEDIMIENTO DERECOLECCIÓN DEDATOS}

La muestra de estudio se dividió en dos grupos, alumnos de cuarto grado y los alumnos de quinto grado.

Se aplicó el inventario de autoestima de Coopersmith a los estudiantes de forma colectiva.

Se aplicó de forma individual y colectiva la Escaia de clima Familiar de Moos a 126 padres de familia.

\section{PROCESAMIENTO DE DATOS}

Con los datos obtenidos calculamos el promedio de desviación estándar, moda y el coeficiente de Pearson. 
Para el cálculo del coeficiente de correlación se empleó las fórmulas correspondientes.

\section{ANÁLISIS}

El análisis y discusión es en base a puntajes de los promedios de desviación estándar, moda del clima familiar de 126 familias encuestadas donde interpretamos la dimensión de la relación familiar, la subescala de cohesión la subescala de expresividad, la subescala de conflicto, la dimensión de crecimiento familiar, la dimensión de mantenimiento del sistema u organización, la nula escala de orientación al logro y la de orientación intelectual.

\section{CONCLUSIONES}

- En la dimensión relación familiar: la sub-escaia cohesión alcanza un puntaje alto. La sub-escala agresividad alcanza lo normal lo cual nos indica que la mayoría de las familias estan compenetradas, se apoyan entre si, se estimulan al actuar libremente y expresar sus sentimientos.

- En la sub-escala de conflicto y agresividad se obtuvo un puntaje bajo es decir que el grado de conflicto y agresividad entre los miembros de la familia es poco explicito.

- En la dimensión Mantenimiento del sistema: se obtuvo un promedio alto, esto indica que las familias en su mayorla le atribuyen gran importancia a la organización y planificación de sus actividades.

- En la dimensión Mantenimiento del sistema: autonomia, orientación al logro, orientación intelectual y moral se obtuvo puntajes que lo clasifican en el nivel normal, es decir que existe un moderado grado de valor a la autosuficiencia, a la toma de decisiones propias al interés por las actividades intelectuales, sociales y culturales.

- En la sub-escala control se obtuvo un valor bajo que significa escaso grado de dirección familiar en función a reglas y procedimientos establecidos.

- En relación a la variable autoestima se concluye que la mayoria de los estudiantes presentan una autoestima normal es decir una moderada aceptación y valoración de sí mismo, se sienten valorados, aceptados y perciben que son importantes tanto en su hogar como en la escuela.

- Los coeficientes de correlación de Pearson entre los puntajes del clima social-familiar y del inventario de autoestima dio como resultado significativas correlaciones, tanto positivas como negativas. De lo que se desprende que a mayor grado de clima socialfamiliar se incrementa también el grado de autoestima de los hijos. Estos resultados nos permiten aceptar las hipótesis propuestas.

\section{XI.SUGERENCIAS}

- Consideramos que los resultados obtenidos en la presente investigación deben ser refrendados con otros a efectos de lograr generalizaciones adecuadas a nuestra realidad.

Es importante señalar la necesidad que tiene el sistema educativo de considerar dentro de sus objetivos la orientación a la familia a fin de posibilitar que el alumno desarrolle y estructure una adecuada autoestima.

\section{XII . REFEREPCIA BIBLIOGRÁFICA}

1. ALLPORT, Gordon. La personalidad. Barcelona; Ed. Perder, 1990.

2. BELTRAN, J. Y BUENO, J. Psicología de la Educación. Mexico, Ed. Alfaomega, 1995.

3. MORA, GUILLERMO Y Otros. Valores Humanos y Actitudes Positivas. Colombia. Ed. McGraw-Hill, 1995.

4. ROSENBERG, M. La autoimagen del adolescente y la sociedad. Buenos Aires, Ed. Paidos, 1973.

5. SATIR, Virginia, kelaciones Humanas en el núcleo familiar. Mexico, Ed. Pax, $; 2 ; 8$ 